\title{
Spectroscopic distances of 28 nearby star candidates ${ }^{\star}$ (Research Note)
}

\author{
H. Jahreiß ${ }^{1}$, H. Meusinger ${ }^{2}$, R.-D. Scholz ${ }^{3}$, and B. Stecklum ${ }^{2}$ \\ 1 Astronomisches Rechen-Institut am Zentrum für Astronomie der Universität Heidelberg, Mönchhofstraße 12-14, \\ 69120 Heidelberg, Germany \\ e-mail: jahreiss@ari.uni-heidelberg.de \\ 2 Thüringer Landesssternwarte Tautenburg, Sternwarte 5, 07778 Tautenburg, Germany \\ 3 Astrophysikalisches Institut Potsdam, An der Sternwarte 16, 14482 Potsdam, Germany \\ Received 28 January 2008 / Accepted 5 March 2008
}

ABSTRACT

\begin{abstract}
Aims. Twenty eight hitherto neglected candidates for the Catalogue of Nearby Stars (CNS) were investigated to verify their classification and to improve their distance estimates. All targets had at least a preliminary status of being nearby dwarf stars based on their large proper motions and relatively faint magnitudes. Better photometric and/or spectroscopic distances were required for selecting stars for further trigonometric parallax measurements.

Methods. Low-resolution spectra were obtained with NASPEC at the Tautenburg $2 \mathrm{~m}$ telescope and with CAFOS at the Calar Alto $2.2 \mathrm{~m}$ telescope. The spectral types of M-type stars were determined by direct comparison of the target's spectra with those of comparison stars of known spectral types observed with the same instrument. The classification of earlier types was performed based on comparison with published spectral libraries.

Results. For most of the target stars reliable spectral types could be determined and in combination with 2MASS photometry new improved distance estimates became available. The majority were classified as $\mathrm{M}$ dwarfs including 11 stars within $25 \mathrm{pc}$. The fainter component of LDS 1365, previously thought to form a nearby common proper motion pair, is according to our results an unrelated high-velocity background star. For several other nearby common proper motion pairs our distance estimates of the fainter components are in good agreement with Hipparcos distances of the brighter components. The three stars in our sample that were previously thought to be white dwarfs (GJ 2091, GJ 2094, GJ 2098) turned out to be more distant high-velocity F- to K-type (sub)dwarfs. For the star with the largest tangential velocity (GJ 2091; $v_{\tan }>500 \mathrm{~km} \mathrm{~s}^{-1}$ ) we have additional evidence for its probable Galactic halo membership from a measured large radial velocity of $266 \pm 25 \mathrm{~km} \mathrm{~s}^{-1}$ and from its $U B V$ photometry indicating a low metallicity.
\end{abstract}

Key words. Galaxy: solar neighborhood - Galaxy: kinematics and dynamics

\section{Introduction}

In our efforts to complete our knowledge on nearby stars, another sample of nearby star candidates was observed at Tautenburg observatory between February 2006 and February 2007. Without exception the candidate list contains high proper motion stars that as far as we know, have not yet been observed spectroscopically. Spectra for these stars were desired not only to complete the CNS (Catalogue of Nearby Stars) data sets but also to obtain first or improved distance estimates.

Most of the candidates were in the CNS database that has been managed by one of us (H.J.) for many years. For example, the three white dwarf candidates originating from Eggen (1968) were already published in the GJ supplement (Gliese \& Jahreiß 1979). No information about the physical properties of these objects could be found in the literature since then.

Furthermore, some faint components of wide common proper motion binaries were included in the observing list. Often the distance information for such stars is taken from the brighter

\footnotetext{
* Based on observations with the $2 \mathrm{~m}$ telescope of the Thüringer Landessternwarte Tautenburg and with the $2.2 \mathrm{~m}$ telescope of the German-Spanish Astronomical Center, Calar Alto, jointly operated by the Max-Planck-Institut für Astronomie Heidelberg and the Instituto de Astrofísica de Andalucía (CSIC).
}

component. Yet, it is desirable to obtain independent distance information for the fainter component.

The remaining late type dwarfs, all of which were selected from LHS, were included in the CNS database only after publication of the preliminary version of the Third Catalogue of Nearby Stars (pCNS3; Gliese \& Jahreiß 1991). They were mostly borderline cases near or just beyond the present search limit of $25 \mathrm{pc}$ for the CNS sample.

\section{Observations and reduction}

Optical spectroscopic data were collected with the Nasmyth Focal Reducer Spectrograph (NASPEC) at the $2 \mathrm{~m}$ telescope of the Thüringer Landessternwarte (TLS) Tautenburg. The V 200 grism was used, which yields a dispersion of $3.4 \AA$ per pixel, or $12 \AA$ FWHM for a slit width of 1 arcsec. The wavelength coverage was from about $4000 \AA$ to $8500 \AA$. For one star (GJ 2091), the Tautenburg spectrum, taken under poor weather conditions, did not have a sufficiently high signalto-noise ratio for a proper classification. Therefore, we applied for director's discretionary time with the $2.2 \mathrm{~m}$ telescope at Calar Alto. The Calar Alto focal reducer and faint object spectrograph CAFOS was used with the B 200 grism giving a wavelength coverage from about $3500 \AA$ to $7000 \AA$ and a dispersion 
Table 1. Nearby candidates with new proper motions and previously known spectral characteristics and parallaxes.

\begin{tabular}{|c|c|c|c|c|c|c|c|c|c|c|c|c|c|c|c|}
\hline \multirow[t]{2}{*}{ cns } & \multicolumn{2}{|r|}{ RA } & \multicolumn{2}{|c|}{ J2000.0 } & \multicolumn{2}{|l|}{ Dec } & \multirow[t]{2}{*}{ Epoch } & \multirow{2}{*}{$\begin{array}{l}\mu \\
\prime \prime / y r\end{array}$} & \multirow{2}{*}{$\Theta$} & \multirow[t]{2}{*}{ SpT } & \multirow[t]{2}{*}{ mag } & \multirow{2}{*}{$\begin{array}{l}\pi \\
\text { mas }\end{array}$} & \multirow[t]{2}{*}{ s.e. } & \multirow[t]{2}{*}{ Source* } & \multirow[t]{2}{*}{ Other } \\
\hline & $\mathrm{h}$ & $\mathrm{m}$ & s & $\circ$ & & ' & & & & & & & & & \\
\hline GJ 1167B & 13 & 09 & 41.96 & +29 & 01 & 56.9 & 2000.277 & 0.292 & 234.9 & $\mathrm{~m}$ & $17.6 \mathrm{P}$ & & & & LDS 1365B \\
\hline GJ 2091 & 12 & 04 & 56.32 & +04 & 19 & 56.0 & 2000.137 & 0.199 & 221.9 & WD? & 15.03 & 83. & 27. & $\mathrm{a}$ & G 13-6 \\
\hline GJ 2098 & 13 & 08 & 28.67 & +40 & 27 & 10.7 & 1999.274 & 0.263 & 242.2 & WD? & 14.00 & 160. & 52. & $\mathrm{a}$ & G 164-46 \\
\hline NN 817 & 09 & 53 & 33.53 & +50 & 45 & 03.8 & 1999.860 & 0.530 & 272.0 & $\mathrm{~m}$ & 12.02 & 39.8 & 3.3 & $\mathrm{~h}$ & LHS 2205 \\
\hline NN 953 & 11 & 22 & 36.29 & +35 & 46 & 19.0 & 1998.189 & 0.185 & 7.3 & $\mathrm{~m}$ & 14.62 & 41.3 & 9.3 & $\mathrm{p}$ & LP 264-44 \\
\hline NN 1047A & 12 & 21 & 27.05 & +30 & 38 & 35.7 & 2000.263 & 0.331 & 216.9 & $\mathrm{~m}$ & 15.31: & 81.7 & 31.3 & $\mathrm{p}$ & G 148-47 \\
\hline NN 1047B & 12 & 21 & 26.73 & +30 & 38 & 37.6 & 2000.263 & 0.331 & 216.9 & $\mathrm{~m}$ & 15.4: & 81.7 & 31.3 & $\mathrm{p}$ & G 148-48 \\
\hline NN 1073 & 12 & 38 & 36.21 & +05 & 21 & 38.5 & 2000.184 & 0.542 & 266.8 & $\mathrm{k}-\mathrm{m}$ & 15.2: & 38.4 & 10.8 & $\mathrm{p}$ & LHS 2593 \\
\hline NN 1083 & 12 & 43 & 47.59 & +00 & 03 & 20.4 & 1999.063 & 0.691 & 224.8 & $\mathrm{~m}$ & 14.67 & 33.0 & 7.6 & $\mathrm{p}$ & LHS 2616 \\
\hline NN 1169 & 13 & 39 & 15.27 & +15 & 40 & 59.5 & 1998.077 & 0.980 & 142.3 & $\mathrm{~m}$ & 14.16: & 34.4 & 5.0 & $\mathrm{t}$ & LHS 2774 \\
\hline NN 1246A & 14 & 28 & 21.52 & +05 & 19 & 01.4 & 2000.263 & 0.376 & 260.6 & g-k & 12.54 & 70.4 & 51.5 & $\mathrm{p}$ & G 65-54 \\
\hline NN 1246B & 14 & 28 & 17.58 & +05 & 18 & 45.9 & 2000.263 & 0.378 & 259.3 & g-k & 13.13 & 70.4 & 51.5 & $\mathrm{p}$ & G $65-53$ \\
\hline NN 1342B & 15 & 35 & 25.67 & +60 & 05 & 07.7 & 1999.348 & 0.231 & 132.9 & $\mathrm{~m}$ & 13.46 & 52.4 & .7 & $\mathrm{~h}$ & LP 99-392 \\
\hline NN 1361A & 15 & 50 & 18.62 & +34 & 37 & 11.5 & 1998.255 & 0.195 & 222.6 & $\mathrm{~m}$ & 13.04: & 37.1 & 8.3 & $\mathrm{p}$ & LP 274-11 \\
\hline NN 1361B & 15 & 50 & 18.55 & +34 & 37 & 15.9 & 1998.255 & 0.195 & 222.6 & $\mathrm{~m}$ & 16.5: & 37.1 & 8.3 & $\mathrm{p}$ & LP 274-10 \\
\hline NN 1538B & 18 & 00 & 45.44 & +29 & 33 & 56.7 & 1999.260 & 0.222 & 321.2 & $\mathrm{k}$ & $14.5 \mathrm{P}$ & 34.6 & .7 & $\mathrm{~h}$ & LP 333-29 \\
\hline nn 40 & 08 & 12 & 26.57 & +43 & 09 & 29.0 & 1998.852 & 0.558 & 165.2 & $\mathrm{k}-\mathrm{m}$ & 14.26 & 38.3 & 8.7 & $\mathrm{p}$ & LHS 1988 \\
\hline nn 44 & 09 & 08 & 50.79 & +54 & 07 & 18.3 & 1999.948 & 0.553 & 221.4 & $\mathrm{~m}$ & 15.04 & 42.3 & 9.1 & $\mathrm{p}$ & LHS 2109 \\
\hline nn 51 & 11 & 00 & 35.69 & +37 & 28 & 50.8 & 1998.271 & 0.724 & 251.8 & $\mathrm{k}-\mathrm{m}$ & 15.36 & 42.9 & 9.1 & $\mathrm{p}$ & LHS 2340 \\
\hline nn 76 & 13 & 32 & 00.07 & +66 & 36 & 00.5 & 1999.263 & 0.567 & 300.5 & $\mathrm{~m}$ & 14.05 & 40.6 & 9.4 & $\mathrm{p}$ & LHS 2748 \\
\hline nn 77 & 14 & 07 & 48.03 & +57 & 11 & 45.3 & 1999.123 & 0.693 & 285.4 & $\mathrm{~m}$ & 14.20 & 47.1 & 10.5 & $\mathrm{p}$ & LHS 2864 \\
\hline nn 78 & 14 & 16 & 42.11 & +36 & 00 & 50.8 & 2000.186 & 0.542 & 146.1 & $\mathrm{k}-\mathrm{m}$ & 12.59 & 33.9 & 9.2 & $\mathrm{p}$ & LHS 2886 \\
\hline nn 79 & 14 & 20 & 39.46 & +46 & 02 & 34.2 & 1998.395 & 0.645 & 253.6 & $\mathrm{~m}$ & 15.10 & 45.4 & 9.6 & $\mathrm{p}$ & LHS 2897 \\
\hline nn 87 & 15 & 03 & 35.01 & +84 & 38 & 26.7 & 1999.296 & 0.578 & 221.0 & $\mathrm{~m}$ & 14.15 & 33.7 & 7.6 & $\mathrm{p}$ & LHS 3017 \\
\hline nn 90 & 16 & 34 & 13.72 & +54 & 23 & 39.8 & 1999.356 & 0.569 & 337.5 & $\mathrm{k}$ & 11.86 & 33.9 & 9.1 & $\mathrm{p}$ & LHS 3214 \\
\hline
\end{tabular}

* a: WD by Eggen (1968), p: photometric parallax, h: Hipparcos (ESA 1997) parallax, and t: ground-based trigonometric parallax.

of $4.7 \AA$ per pixel. All spectra were reduced with standard routines from the ESO MIDAS data reduction package. For details of the data reduction we refer to our previous paper (Scholz et al. (2005).

The list of nearby star candidates observed in Tautenburg during four runs between February 2006 and February 2007 is presented in Table 1, with an internal CNS designation in Col. 1, the 2MASS right ascension and declination for equinox J2000.0 in Cols. 2 and 3, respectively, and the epoch of the 2MASS observation in Col. 4. The annual proper motion and its direction is given in Cols. 5 and 6 . These proper motions were all new determinations making use of the available new digitized sky surveys like DSS and 2MASS All our objects are at northern declinations, therefore epoch differences of up to 50 years or more became available with the USNO A2 positions from the first Palomar Observatory Sky Survey (POSS-I) leading to proper motion errors well below $10 \mathrm{mas} / \mathrm{yr}$. In Col. 7 the previously known information about spectral characteristics is given, i.e. mostly spectral classes from Luyten's proper motion surveys. In Col. 8 visual or photographic (followed by a P) magnitudes are listed. In Cols. 9 and 10 the previously known parallax and its standard error are given, and in Col. 11 the source code for this parallax. An additional identifier given in the last column should allow easier access to the Simbad database for further information.

Additional spectra of nine stars with known spectral types from M0 to M6.5 (see Fig. 1) were taken to allow a direct comparison of our target spectra. Unfortunately, we could not make use of the spectrum taken for the M2 dwarf Gl 38. Therefore a comparison spectrum for M2 dwarfs could not be performed. For the M dwarf stars the spectral type was then estimated by comparing the spectra in question directly with the sequence of comparison spectra. All spectra were thus normalized at $7500 \AA$. Figure 1 shows as an example the spectrum of GJ 1167B compared to the spectra of eight comparison stars. Visual inspection allows a rather accurate determination of the spectral type. However, the calibration of the spectra below $5000 \AA$ and above $8000 \AA$ is less certain.

In addition to the visual estimate an automatic classification method was applied. The difference between the spectrum of the object and each comparison spectrum was calculated and the variance was used as a measure of agreement. The best fit spectral type was then obtained by searching for the minimum of the variance as a function of spectral type using a quadratic regression. Generally, the automatic method provided results in good agreement with the visual classification. Nevertheless, we preferred the results of the latter method.

\section{Object classification and distance estimates}

In Table 2 the finally adopted spectral types are given in Col. 2. For all the M dwarfs, these spectral types were obtained by visual inspection, comparing the target spectra with the sequence of comparison spectra. Column 3 lists spectral types obtained by the automatic method described in Sect. 2. The adopted spectral types in Col. 2 are followed by the letter "e" if an $\mathrm{H} \alpha$ emission line was detected. It is interesting that all emission line $\mathrm{M}$ dwarfs were also identified as X-ray sources according to the VizieR Catalogue Service (http://vizier.u-strasbg. $\mathrm{fr} /$ ). The 2MASS magnitude $J$ in Col. 4 was used to compute the spectroscopic parallaxes listed in Col. 5 with the recently 


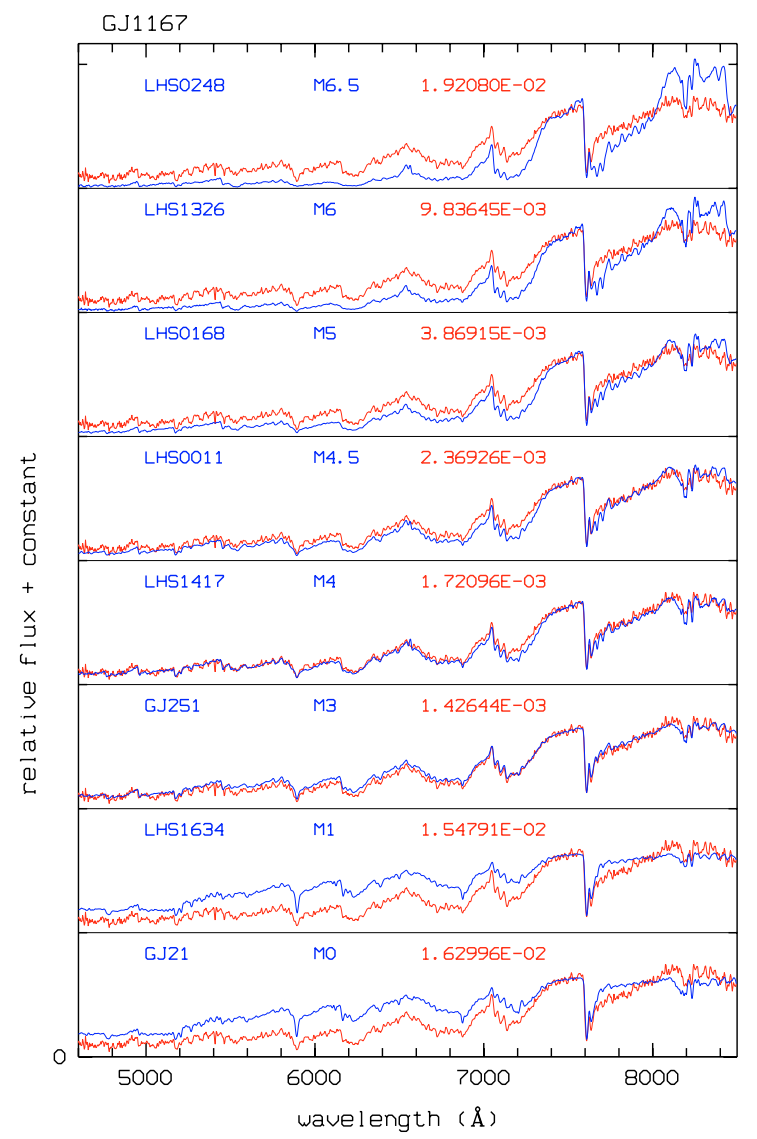

Fig. 1. Spectrum of GJ 1167B in comparison to a sequence of M dwarfs with known spectral types. The numbers give the variance between the target spectrum and a given comparison spectrum. The signal-to-noise of the NASPEC spectra shown here are typical of all our M-type targets.

calibrated spectral type vs. $M_{J}$ relation for late type dwarf stars given in Table 2 of Scholz et al. (2005).

The spectra of the three previously suspected white dwarfs GJ 2091, GJ 2094, and GJ 2098 are shown in Figs. 2-4, respectively. These are clearly not white dwarf spectra but rather are typical for F- to K-type dwarfs, although in the case of GJ 2091 we were initially not sure. The NASPEC spectrum of this object (not shown here) was very noisy allowing only the identification of the $\mathrm{H} \alpha$ and $\mathrm{H} \beta$ lines. But no other, even broad stellar features could be seen. However, the higher-quality CAFOS spectrum (Fig. 2) ruled out a white dwarf. The signal-to-noise ratio of the NASPEC spectrum of GJ 2094 (Fig. 3) is as low as in the poor NASPEC spectrum of GJ 2091, but broad absorption to the blue side of the $\mathrm{MgI}$ line (due to $\mathrm{MgH}$ ) and a strong $\mathrm{NaI}$ line are clearly seen in addition to $\mathrm{H} \alpha$ and $\mathrm{H} \beta$.

For our objects of earlier spectral types $(<\mathrm{M})$ we had no comparison objects observed with the same instrument. In order to classify them we searched for similar spectra in the spectral libraries of Torres-Dodgen \& Weaver (1993) and Jacoby et al. (1984). Based on the strong $\mathrm{NaI}$ and weak $\mathrm{H} \alpha$ we classify GJ 2094 as a mid-K dwarf. It could well be a subdwarf since the spectrum looks very similar to that of the high proper motion star SSSPM J1549-3544 classified as sdK5 by Farihi et al. (2005). In the case of GJ 2098 the $\mathrm{NaI}$ line is much weaker compared to $\mathrm{H} \alpha$, which is typical for $\mathrm{G}$ dwarfs. The strong Balmer lines together with rather weak $\mathrm{MgI}$ and $\mathrm{NaI}$ lines suggest an even earlier, F-type classification for GJ 2091.
Table 2. New spectral types, 2MASS photometry, and resulting spectroscopic parallaxes.

\begin{tabular}{lccrrl}
\hline \hline cns & SpT & SpT $_{\mathrm{c}}$ & \multicolumn{1}{c}{$J$} & $\pi_{\mathrm{sp}}$ & other \\
\hline GJ 1167B & M3.5 & M3.4 & 13.733 & 6.5 & LDS 1365B \\
GJ 2091 & $<$ (sd)G0 & & 13.667 & $<1.8$ & G 13-6 \\
GJ 2094 & $\sim$ (sd)K5 & & 14.406 & $<2.9$ & GD 266 \\
GJ 2098 & $<$ (sd)K0 & & 12.405 & $<4.9$ & G 164-46 \\
NN 817 & M1.5 & M1.5 & 8.733 & 37.8 & LHS 2205 \\
NN 953 & M4.0 & M3.7 & 10.243 & 42.2 & LP 264-44 \\
NN 991 & M3.0 & M2.8 & 10.406 & 24.7 & LP 433-54 \\
NN 1047A & M4.5e & M3.9 & 9.987 & 59.2 & G 148-47 \\
NN 1047B & M5.0e & M6.1 & 10.057 & 70.6 & G 148-48 \\
NN 1073 & M4.5 & M4.5 & 10.768 & 41.3 & LHS 2593 \\
NN 1083 & M4.0 & M3.2 & 10.420 & 38.9 & LHS 2616 \\
NN 1169 & M3.5 & M3.4 & 10.333 & 31.3 & LHS 2774 \\
NN 1246A & M3.5 & M3.3 & 8.724 & 65.6 & G 65-54 \\
NN 1246B & M3.0 & M2.4 & 8.932 & 48.7 & G 65-53 \\
NN 1342B & M4.0e & M3.4 & 9.270 & 66.1 & LP 99-392 \\
NN 1356 & M3.0 & M2.6 & 9.464 & 38.1 & LHS 3123 \\
NN 1361A & M3.0 & M2.8 & 9.414 & 39.0 & LP 274-11 \\
NN 1361B & M4.5e & M4.4 & 11.025 & 36.7 & LP 274-10 \\
NN 1538B & M2.0 & M1.9 & 9.058 & 36.8 & LP 333-29 \\
nn 40 & M3.5 & M2.6 & 9.994 & 36.6 & LHS 1988 \\
nn 44 & M4.0 & M3.0 & 10.530 & 37.0 & LHS 2109 \\
nn 51 & M4.5 & M4.4 & 10.659 & 43.5 & LHS 2340 \\
nn 76 & M3.5 & M3.6 & 9.916 & 37.9 & LHS 2748 \\
nn 77 & M4.0 & M3.2 & 9.875 & 50.0 & LHS 2864 \\
nn 78 & M2.0 & M2.0 & 9.183 & 34.8 & LHS 2886 \\
nn 79 & M4.5 & M4.3 & 10.455 & 47.8 & LHS 2897 \\
nn 87 & M4.0 & M3.2 & 10.071 & 45.7 & LHS 3017 \\
nn 90 & M0.0 & M1.1 & 8.720 & 29.0 & LHS 3214 \\
\hline & & & & &
\end{tabular}

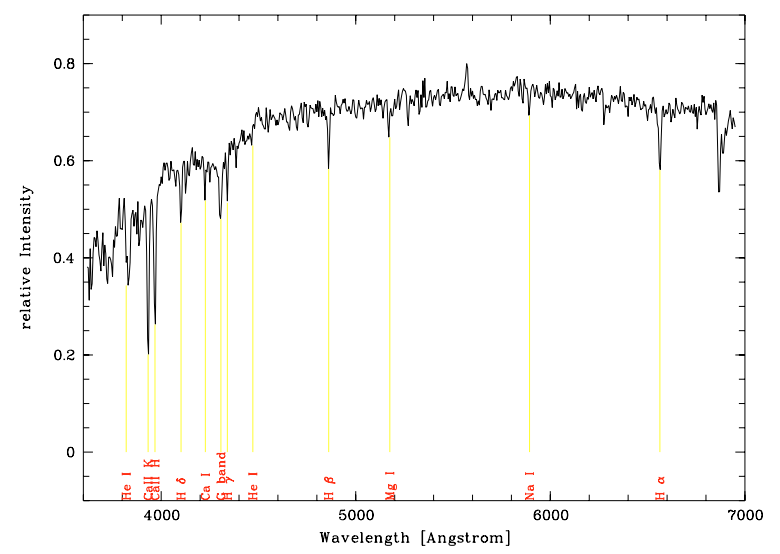

Fig. 2. CAFOS B200 spectrum of GJ 2091.

If we assume all three stars GJ 2094, GJ 2098, and GJ 2091 to be on the main sequence and adopt a rough classification as $\sim \mathrm{K} 5,<\mathrm{K} 0$, and $<\mathrm{G} 0$, we obtain very large distances of $680 \mathrm{pc}$, $>410 \mathrm{pc}$, and $>1100 \mathrm{pc}$, respectively, again by using the absolute $J$ magnitudes for K5 and K0 stars from Scholz et al. (2005) and an $M_{J}=3.4$ for a G0 star (Boccaletti et al. 2003). Consequently, these distances lead to extremely high tangential velocities of $450 \mathrm{~km} \mathrm{~s}^{-1},>500 \mathrm{~km} \mathrm{~s}^{-1}$, and $>1000 \mathrm{~km} \mathrm{~s}^{-1}$, respectively. If all three objects were subdwarfs, their distances and tangential velocities would be reduced by roughly $50 \%$ (Scholz et al. 2005). All three objects are far beyond the Solar neighbourhood and possess very high velocities typical for the Galactic halo population. 


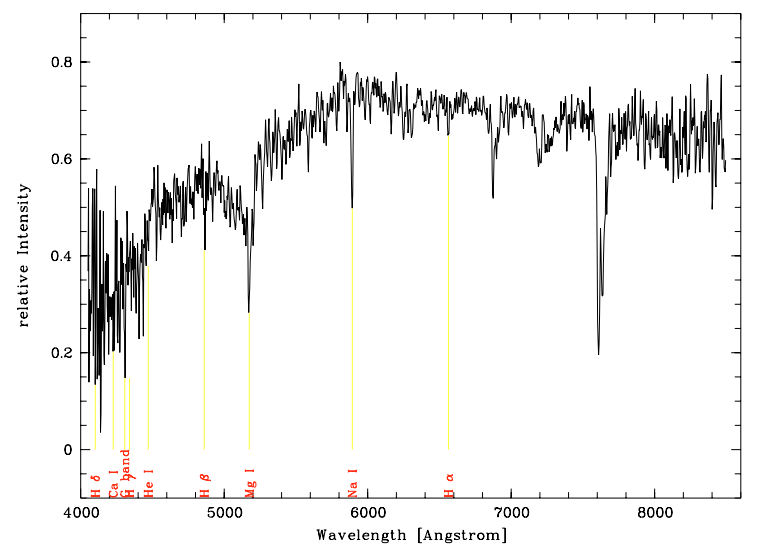

Fig. 3. NASPEC V200 spectrum of GJ 2094.

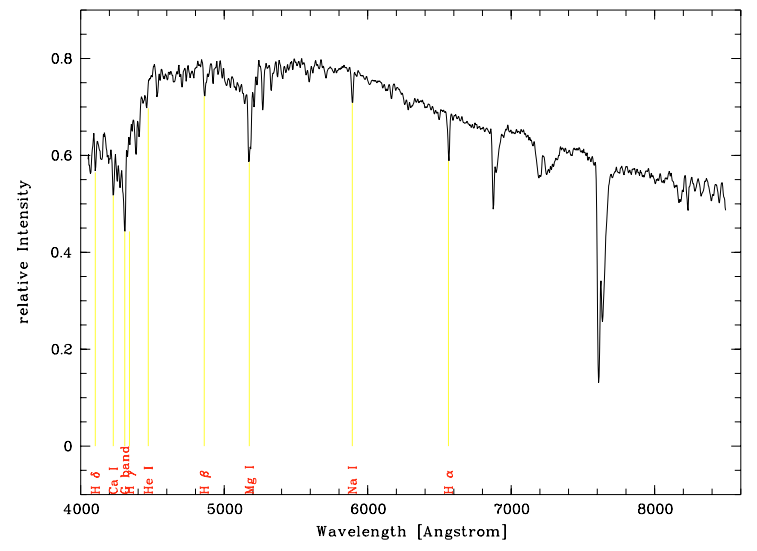

Fig. 4. NASPEC V200 spectrum of GJ 2098.

\section{Individual objects}

In the following some of the most interesting objects are discussed in more detail.

\subsection{GJ 1167B}

In his second list of common proper motion pairs Luyten (1969) reported LDS 1365 with a common proper motion of $0.39^{\prime \prime} / \mathrm{yr}$ in $240^{\circ}$ and a large separation of $190^{\prime \prime}$ in $26^{\circ}$. A spectral class " $\mathrm{m}$ " was assigned to both components. In a subsequent publication Luyten (1973) designated the brighter component as LP 322-836 and the fainter one as LP 322-835. The couple was included in the GJ-supplement as GJ 1167A and B on the basis of a photometric parallax of 54 mas for the brighter star LP 322-836. A first trigonometric parallax from Sproul Observatory (Heintz 1994) $\pi=86 \pm 12.5$ mas moved it even closer to the Sun. This value was recently confirmed by Smart et al. (2007) who determined the trigonometric parallax of LP 322-836 to $\pi=83.1 \pm 6.2$ mas.

Sanduleak's (1976) remark (see star No. 224) that he found a much brighter non-M star at Luyten's position for LP 322-835 may have caused Luyten to omit LP 322-835 from his NLTTcatalog, and so it was also excluded from the pCNS3.

New proper motion determinations based on the modern DSS etc. yield $\mu=0.398^{\prime \prime} / \mathrm{yr}$ in $239^{\circ} .1$ for LP 322-836 and $\mu=$ $0.291^{\prime \prime} / \mathrm{yr}$ in $234^{\circ} 8$ for LP 322-835, arguing against a physical connection. Now our spectral type M3 for LP 322-835 allowed a first direct distance determination for component B as $190 \mathrm{pc}$.
This is in close agreement with a distance of $175 \mathrm{pc}$ which we derived simultaneously from Sloan colours $i-z$ applying the calibration given in Hawley et al. (2002). Thus, LDS 1365 does not form a common proper motion pair. LP 322-836 - henceforth GJ 1167 - is a nearby slow moving young M4 dwarf showing $\mathrm{X}$-ray emission. On the other hand the formerly supposed common proper motion companion LP 322-835 is a distant M3 dwarf with a rather high $\left(\sim 250 \mathrm{~km} \mathrm{~s}^{-1}\right)$ tangential velocity.

\subsection{GJ 2091}

In an investigation of faint blue stars, Eggen (1968) suspected this high proper motion star to be a white dwarf. On the basis of his $U B V$ photometry he deduced a parallax of 83 mas in applying the $U-V, M_{V}$ relation. With this information G 13-6 was included in Table 2 (Suspected Nearby Stars) of the GJ-supplement Gliese \& Jahreiß (1979). No further results have been published. This applies also to the two stars GJ 2094 and GJ 2098, with a similar history.

The NASPEC spectrum of GJ 2091 was obtained under poor weather conditions, and led us to a preliminary classification of this object as a cool DA white dwarf with a clear indication of $\mathrm{H} \alpha$ and $\mathrm{H} \beta$ absorption lines and no further significant features. In this case GJ 2091 would have a distance close to 10 parsec.

Yet, for a definitive conclusion a better spectrum was required, and we applied for DDT service observation with CAFOS at the $2.2 \mathrm{~m}$ telescope at Calar Alto. Such an observation was successfully carried out on April 23, 2007. The analysis of the spectrum (Fig. 2) shows clearly that GJ 2091 is not a white dwarf but rather an F-G dwarf star so that it can definitively be excluded from the CNS. Nevertheless we determined a radial velocity for this object, based on measurements of the four Balmer lines $\mathrm{H} \alpha, \ldots, \mathrm{H} \delta$ with respect to the spectrum of the standard star, Feige 66, observed during the same night. The radial velocity was $+266 \pm 25 \mathrm{~km} \mathrm{~s}^{-1}$, a rather large value, i.e. GJ 2091 is really a fast moving high velocity star, probably a member of the Galactic halo. The available $U B V$ photometry of GJ 2091 indicates a low metallicity $[\mathrm{Fe} / \mathrm{H}]=-2.4$ according to Carney (1979), which is also an indication of membership in the Galactic halo.

\section{3. $L H S 2205=N N 817$}

With a Hipparcos parallax of $39.75 \pm 3.27$ mas, LHS 2205 is very close to the search limit of CNS. It was missed by former spectroscopic surveys due to its slightly smaller photometric parallax of 38 mas, the reason for its exclusion from the pCNS3. Its new spectral type M1.5 yields a spectroscopic parallax of 38 mas, in full agreement with the photometric value and the trigonometric parallax measured by Hipparcos.

\section{4. $L P 433-54=N N 991$}

Optical photometry yields a distance of $24 \mathrm{pc}$, whereas infrared photometry as well as the new M3.0 spectral type determined predict a considerably larger distance of up to $40 \mathrm{pc}$.

\section{5. $G 148-47 / 48=N N 1047 A B$}

A first distance of 14 pc for this close pair (separation 4.5") was determined by Pesch \& Dahn (1982) calling it Sanduleak $57+58$. For their estimate the authors used VI photometry, $V=14.51, V-I=3.40$ where the $V-I$ was measured 
in the Kron-Mayall system. Transforming this value to the Kron-Cousins system according to Leggett (1992) yields only a minor change to $V-I=3.39$. Fleming (1998) published an independent distance estimate of $11.2 \mathrm{pc}$ from his slightly different $V I$ photometry (in the Kron-Cousins system) $V=14.709, V-I=3.465$ without being aware that it is a close pair. The latter was recently discussed by Reid et al. (2004), who obtained a revised distance estimate for the system of $\sim 12.5 \mathrm{pc}$.

Averaging our independent spectroscopic distance estimates for the two components we obtain a mean distance of $17 \mathrm{pc}$ for the system. This is close to the corrected value of $15.8 \mathrm{pc}$ from the original estimate of Fleming (1998) assuming a system of almost equal brightness components, which is supported by several observations.

\section{6. $G 65-54 / 53=N N 1246 A B$}

This common proper motion pair separated by 61 arcsec had up to now only $U B V$-photometry available with $B-V>1.50$ for both components. Because a value of $B-V \geq 1.30$ does not allow a reasonable distance estimate, this pair was excluded from the pCNS3. Now with the new spectral types and 2MASS photometry a distance of $17.5 \mathrm{pc}$ is proposed.

\section{7. $L P 99-392=N N 1342 B$}

LP 99-392 is the fainter common proper motion companion (separation $43^{\prime \prime}$ ) to HD 139477 . Here we had the curious case that a VRI-photometric parallax of 55 mas was available for the fainter component, whereas for the brighter component only the HD-type K5 was known. Therefore the pair was not included in the pCNS3 although already then it was present in the list of nearby candidates.

Only in 1997 did Hipparcos determine a distance of 19 pc for HD 139477, and almost simultaneously an MK spectral type of K3 V was published for it (Loth \& Bidelman 1998). The combination of photometric and spectroscopic parallaxes yields a distance of 18 pc for LP 99-392, and also its proper motion of $\mu=0.231^{\prime \prime} / \mathrm{yr}$ in $132^{\circ} .9$ is in good agreement with the Hipparcos value $\mu=0.2370^{\prime \prime} / \mathrm{yr}$ in $133^{\circ} .4$ for HD 139477 . In other words the physical connection of this common proper motion pair is now well established.

\section{8. $L P 333-29=N N 1538 B$}

Already the newly determined proper motion $\mu=0.222^{\prime \prime} / \mathrm{yr}$ in 321.8 for LP 333-29 allowed little doubt about the physical relation with the G2 V primary HD 164595 about $88^{\prime \prime}$ away having a Hipparcos proper motion of $\mu=0.2224^{\prime \prime} / \mathrm{yr}$ in $321^{\circ} .2$. Now our spectral type of M2.0 provides a direct distance estimate for LP 333-29 as 27.2 pc, which is in almost perfect agreement with the Hipparcos parallax of 34.57 mas for the primary, and the metallicity $[\mathrm{Fe} / \mathrm{H}]=-0.07$ determined for the primary in Bonfils et al. (2005) applies also to the M2 dwarf LP 333-39.

\subsection{LHS $1988=n n 44$}

VRI photometry consistently provides a parallax of 42 mas. $V-K_{\mathrm{s}}$ and the new spectral type M4.0 yield slightly smaller parallax values of 38 mas, and 37 mas, respectively.

\section{Conclusions}

With the spectroscopic distances determined in this paper, we were able to solve several doubtful distance estimates for nearby star candidates. Among these Eggen's white dwarf candidates contained in the GJ-supplement are all far away F-K dwarf stars. From their extremely high tangential velocities we suspect them to be subdwarfs. With this classification they would still have large velocities typical of Galactic halo stars. Our measured radial velocity for the star with the highest tangential velocity, GJ 2091, confirms this assumption.

All other stars in our sample were classified as M dwarfs. According to our new distance estimates all but one of these 25 stars lie within $40 \mathrm{pc}$, including 11 objects falling within the 25 pc horizon of the CNS.

We could show that Luyten's common proper motion pair LDS 1365 consists of two M dwarf stars with similar proper motions but completely different distances and space velocities.

Our spectroscopy in combination with 2MASS colours provided the first reliable distance estimate for the common proper motion pair G65-54/53, which is probably within 20 pc.

Acknowledgements. We thank the Calar Alto Observatory for allocation of director's discretionary time to this programme. We acknowledge the use of the Simbad database, the VizieR Catalogue Service operated at the CDS, and the use of the 2MASS All-Sky Survey.

\section{References}

Boccaletti, A., Chauvin, G., Lagrange, A.-M., \& Marchis, F. 2003, A\&A, 410, 283

Bonfils, X., Delfosse, X., Udry, S., et al. 2005, A\&A, 442, 635

Carney, B. W. 1979, ApJ, 233, 211

Eggen, O. J. 1969, ApJS, 16, 97

ESA 1997, The Hipparcos and Tycho Catalogues, ESA SP-1200

Farihi, J., Wood, P. R., \& Stalder, B. 2005, ApJ, 627, L41

Fleming, Th. A. 1998, ApJ, 504, 461

Gliese, W., \& Jahreiß, H. 1979, A\&AS, 38, 423

Gliese, W., \& Jahreiß, H. 1991, Preliminary Version of the Third Catalogue of Nearby Stars, on ADS CD-ROM, 1

Hawley, S. L., Covey, K. R., Knapp, G. R., et al. 2002, AJ, 123, 3409

Heintz, W. D. 1994, AJ, 108, 2338

Jacoby, G. H., Hunter, D. A., \& Christian, C. A. 1984, ApJS, 56, 257

Leggett, S. K. 1992, ApJS, 82, 351

Lot, A. L., \& Bidelman, W. P. 1998, PASP, 110, 268

Luyten, W. J. 1969, Proper motion survey with the 48-inch Schmidt telescope, Vol. XXI, Univ. of Minnesota, Minneapolis

Luyten, W. J. 1973, Proper motion survey with the 48-inch Schmidt telescope,

Vol. XXXV, Univ. of Minnesota, Minneapolis

Pesch, P., \& Dahn, C. 1982, AJ, 87, 122

Reid, I. N., Cruz, K. L., Allen, P., et al. 2004, AJ, 128, 463

Sanduleak, N. 1976, AJ, 81, 350

Scholz, R.-D., Meusinger, H., \& Jahreiß, H. 2005, A\&A, 442, 211

Smart, R. L., Lattanzi, M. G., Jahreiß, H., et al. 2007, A\&A, 464, 787

Torres-Dodgen, A. V., \& Weaver, W. B. 1993, PASP, 105, 693 